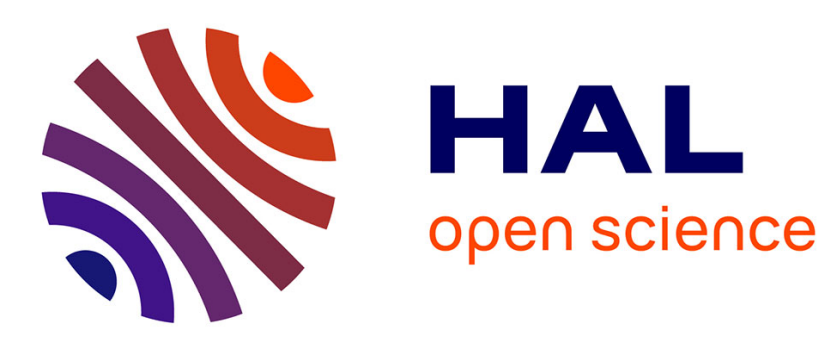

\title{
New Fixed Time and Fast Converging Reduced Order Observers
}

\author{
Frederic Mazenc, Michael Malisoff
}

\section{To cite this version:}

Frederic Mazenc, Michael Malisoff. New Fixed Time and Fast Converging Reduced Order Observers. CDC2021 - Conference on Decision and Control, Dec 2021, Austin, United States. hal-03431462

\section{HAL Id: hal-03431462 \\ https://hal.inria.fr/hal-03431462}

Submitted on 16 Nov 2021

HAL is a multi-disciplinary open access archive for the deposit and dissemination of scientific research documents, whether they are published or not. The documents may come from teaching and research institutions in France or abroad, or from public or private research centers.
L'archive ouverte pluridisciplinaire HAL, est destinée au dépôt et à la diffusion de documents scientifiques de niveau recherche, publiés ou non, émanant des établissements d'enseignement et de recherche français ou étrangers, des laboratoires publics ou privés. 


\title{
New Fixed Time and Fast Converging Reduced Order Observers
}

\author{
Frédéric Mazenc and Michael Malisoff
}

\begin{abstract}
For nonlinear continuous-time systems with continuous measurements of the output, we provide new reduced order observers that converge in finite time. The convergence time is independent of the initial state. For cases where the measurements are discrete, we provide asymptotically converging observers, whose rate of convergence is proportional to the negative of the logarithm of the size of the sampling interval. Our observers are based on the observability Gramian.
\end{abstract}

\section{INTRODUCTION}

As explained in [11], finite time observers offer considerable promise for an ever-growing range of practical applications, because of their ability to compute exact values of states in a finite time, and many contributions are devoted to the design of observers of this type. Some of them use delays, dynamic extensions, homogenous functions, sliding mode, or unbounded gains; see for instance, [2], [3], [5], [7], [14], and [17]. The works [11] and [15] are different because they use observers with impulses and no delay.

In this paper, we continue our work on the design of fixed time observers. Fixed time convergence means that the convergence time is independent of the initial state. This differs from semi-global works such as [18] whose finite convergence time depends on the initial state. As in [11], we provide new reduced order observers for continuous-time nonlinear systems, first when there are continuous output measurements and next in the case where there are only discrete output measurements. When continuous measurements are available, we provide observers that converge in finite time. When only discrete measurements are available, we provide observers that do not converge in finite time, but which do converge asymptotically with a rate of convergence that is proportional to the negative of the logarithm of the size of the sampling interval. This ensures arbitrarily fast convergence, by picking the sampling interval small enough.

The fundamental difference between [11] and the present paper is that the observer we introduce is not based on the one proposed in [15]. Instead, the observer we propose here uses discrete variables and the observability Gramian and the solutions of the observer are continuous. It is also very different from those of [1], [4], [9], [12], and [16], which are based on observers with delays. By not using the delays that occur in earlier observer designs, we obtain simpler reduced order controllers that still enjoy the required fixed

Key Words: Reduced order observer, finite time, discrete measurements. Supported by NSF Grants 1711299 and 2009659 (Malisoff).

F. Mazenc is with Inria Saclay, L2S-CNRS-CentraleSupélec 3 rue Joliot Curie, 91192, Gif-sur-Yvette, France (e-mail: frederic.mazenc@12s.centralesupelec.fr)

M. Malisoff is with Department of Mathematics, Louisiana State University, Baton Rouge, LA 70803, USA (e-mail: malisoff@1su.edu) time or arbitrarily fast convergence of the observation error to zero. Our convergence proof for our second observer uses the trajectory based approach, which was introduced in [10] and developed in several papers such as [13].

The paper is organized as follows. Our observer result under continuous measurements is stated and proven in Section II, and Section III provides our analog for cases where only discrete measurements are available. We illustrate our approach in Section IV, using a pendulum example. We summarize our results and our suggestions for further research in Section V.

Notation. We use standard notation, which we simplify when no confusion would arise. The dimensions of our Euclidean spaces are arbitrary, unless we indicate otherwise. The standard Euclidean 2-norm, and its induced matrix norm, are denoted by $|\cdot|,|\cdot|_{S}$ denotes the essential supremum over any set $S, \mathbb{N}=\{1,2, \ldots\}$, and $\mathbb{Z}_{\geq 0}=\{0\} \cup \mathbb{N}$. We let $I$ denote the identity matrix of any dimension.

\section{OBSERVERS FOR CONTINUOUS MEASUREMENTS}

\section{A. Statement of Result}

We consider the system

$$
\left\{\begin{aligned}
\dot{\xi}(t) & =\mathcal{A} \xi(t)+\mathcal{F}(\mathcal{C} \xi(t), u(t))+\kappa(t) \\
Y(t) & =\mathcal{C} \xi(t)+\epsilon(t)
\end{aligned}\right.
$$

where $\xi$ is valued in $\mathbb{R}^{n}$, the piecewise continuous locally bounded function $u$ is valued in $\mathbb{R}^{p}$, the output $Y$ is valued in $\mathbb{R}^{q}, \kappa$ and $\epsilon$ are locally bounded and piecewise continuous and represent disturbances, and $\mathcal{F}$ is a nonlinear locally Lipschitz function such that (1) is forward complete. Assume:

Assumption 1: The pair $(\mathcal{A}, \mathcal{C})$ is observable.

Then (e.g. from [8, pp. 304-306]) with an appropriate decomposition of the state vector $\xi$, we obtain

$$
\left\{\begin{array}{l}
\dot{\xi}_{1}(t)=A_{1} \xi_{1}(t)+F_{1}\left(\xi_{2}(t), u(t)\right)+\kappa_{1}(t) \\
\dot{\xi}_{2}(t)=A_{2} \xi_{1}(t)+F_{2}\left(\xi_{2}(t), u(t)\right)+\kappa_{2}(t) \\
Y(t)=\xi_{2}(t)+\epsilon(t)
\end{array}\right.
$$

where $F_{1}$ and $F_{2}$ are locally Lipschitz functions, and where the pair $\left(A_{1}, A_{2}\right)$ is observable. By a change of coordinates, we can assume that $A_{1} \in \mathbb{R}^{(n-q) \times(n-q)}$ is an invertible matrix. (If $A_{1}$ were not invertible, then we could replace it by a new one $A_{2}+L A_{2}$ where $L$ is such that $A_{2}+L A_{2}$ is Hurwitz, by applying the change of coordinates $\xi_{3}(t)=$ $\xi_{1}(t)+L \xi_{2}(t)$, using the fact that if $\left(A_{1}, A_{2}\right)$ is observable then so is $\left(A_{1}+L A_{2}, A_{2}\right)$.) Observability of $\left(A_{1}, A_{2}\right)$ and the invertibility of $A_{1}$ imply that for any constant $\nu>0$ and with the choice

$$
H=A_{2} A_{1}^{-1} \in \mathbb{R}^{q \times(n-q)},
$$


the $(n-q) \times(n-q)$ matrix

$$
W=\left(\int_{0}^{\nu}\left(e^{A_{1}^{\top} \ell}-I\right) H^{\top} H\left(e^{A_{1} \ell}-I\right) \mathrm{d} \ell\right)^{-1}
$$

is invertible. This follows from the fact that if there were a nonzero vector $V$ such that $H\left(e^{A_{1} \ell}-I\right) V=0$ for all $\ell \in[0, \nu]$ then all of the derivatives of $H\left(e^{A_{1} \ell}-I\right) V$ with respect to $\ell$ are equal to zero, which implies that $H A_{1} V=0$, $H A_{1}^{2} V=0, \ldots, H A_{1}^{n} V=0$, which yields a contradiction with the fact that $\left(A_{1}, A_{2}\right)$ is observable.

Let $\alpha: \mathbb{R} \rightarrow \mathbb{R}^{(n-q) \times q}$ be defined by

$$
\alpha(\ell)=W\left(e^{A_{1}^{\top} \ell}-I\right) H^{\top} .
$$

Let $t_{i}=i \nu$ for all $i \in \mathbb{Z}_{\geq 0}$ and $\sigma$ be the function defined by $\sigma(t)=t_{i}$ when $t \in\left[t_{i}, t_{i+1}\right)$. We define $\zeta: \mathbb{R} \rightarrow \mathbb{R}^{n-q}$ by

$$
\begin{aligned}
& \zeta(t)=\int_{\sigma(t)}^{t} e^{A_{1}(t-s)} \kappa_{1}^{\sharp}(s) \mathrm{d} s \\
& -e^{A_{1}(t-\sigma(t))} \int_{\sigma(t)}^{t} \alpha(s-\sigma(t))\left[\int_{\sigma(t)}^{s} \kappa_{2}^{\sharp}(\ell) \mathrm{d} \ell\right. \\
& \left.+A_{2} \int_{\sigma(t)}^{s} \int_{\sigma(t)}^{m} e^{A_{1}(m-\ell)} \kappa_{1}^{\sharp}(\ell) \mathrm{d} \ell \mathrm{d} m\right] \mathrm{d} s \\
& -e^{A_{1}(t-\sigma(t))} \int_{\sigma(t)}^{t} \alpha(s-\sigma(t))(\epsilon(s)-\epsilon(\sigma(t))) \mathrm{d} s
\end{aligned}
$$

where for $i=1$ and 2 ,

$$
\begin{aligned}
& \kappa_{i}^{\sharp}=\kappa_{i}+\Delta_{i} \text { and } \\
& \Delta_{i}(t)=F_{i}\left(\xi_{2}(t), u(t)\right)-F_{i}(Y(t), u(t)) .
\end{aligned}
$$

We propose a candidate observer:

$$
\left\{\begin{aligned}
\dot{\xi}_{\star, 1}(t)= & A_{1} \xi_{\star, 1}(t)+F_{1}(Y(t), u(t)) \\
\dot{\xi}_{\star, 2}(t)= & A_{2} \xi_{\star, 1}(t)+F_{2}(Y(t), u(t)) \\
\dot{\hat{x}}_{1}(t)= & A_{1} \hat{x}_{1}(t)+e^{A_{1}\left(t-t_{i}\right)} \alpha\left(t-t_{i}\right)[Y(t) \\
& -\xi_{\star, 2}(t)-Y\left(t_{i}\right)+\xi_{\star, 2}\left(t_{i}\right) \\
& \left.-H\left(e^{A_{1}\left(t-t_{i}\right)}-I\right) \hat{x}_{1}\left(t_{i}\right)\right], \\
& \text { for all } t \in\left[t_{i}, t_{i+1}\right) \text { and } i \in \mathbb{Z}_{\geq 0},
\end{aligned}\right.
$$

whose first two equations are solved for all $t \geq 0$, and whose last equation is solved successively on the intervals $\left[t_{i}, t_{i+1}\right)$ for all $i \in \mathbb{Z}_{\geq 0}$ with the initial state $\hat{x}_{1}\left(t_{0}\right)=0$ at time $t_{0}=0$ and the initial states for $\hat{x}_{1}$ at the times $t_{i}$ for $i \geq 1$ given by the left limits $\hat{x}_{1}\left(t_{i}^{-}\right)$for $i \geq 1$ (which we write more concisely as $\hat{x}_{1}\left(t_{i}\right)$ in our observer formula).

We are ready to state and prove the following result:

Theorem 1: Let Assumption 1 hold. Then for all initial states of (2), the solutions of (2) and (8) are such that

$$
\xi_{1}(t)=\hat{x}_{1}(t)+\xi_{\star, 1}(t)+\zeta(t)
$$

for all $t \geq \nu$, where $\zeta$ is defined by (6).

Remark 1: In order to describe how precisely $\hat{x}_{1}(t)+$ $\xi_{\star, 1}(t)$ estimates $\xi_{1}(t)$, let us define the constant

$$
\bar{\alpha}=|\alpha|_{[0, \nu]}
$$

and observe that

$$
\begin{aligned}
|\zeta(t)| \leq & \nu e^{\left|A_{1}\right| \nu \bar{\alpha}}\left[\int_{\sigma(t)}^{t}\left|\kappa_{2}^{\sharp}(\ell)\right| \mathrm{d} \ell\right. \\
& \left.+\nu\left|A_{2}\right| e^{\nu\left|A_{1}\right|} \int_{\sigma(t)}^{t}\left|\kappa_{1}^{\sharp}(\ell)\right| \mathrm{d} \ell\right] \mathrm{d} s \\
& +e^{\nu\left|A_{1}\right|} \int_{\sigma(t)}^{t}\left|\kappa_{1}^{\sharp}(\ell)\right| \mathrm{d} \ell \\
& +e^{\nu\left|A_{1}\right|} \bar{\alpha} \int_{\sigma(t)}^{t}|\epsilon(s)-\epsilon(\sigma(t))| \mathrm{d} s
\end{aligned}
$$

for all $t \geq 0$. Consequently, if there are constants $K_{i} \geq 0$ that satisfy the requirements from Assumption 2, then

$$
\begin{aligned}
& |\zeta(t)| \leq \bar{\zeta}_{1} \int_{\sigma(t)}^{t}\left(\left|\kappa_{1}(\ell)\right|+\left|\kappa_{2}(\ell)\right|\right) \mathrm{d} \ell+\bar{\zeta}_{2} \int_{\sigma(t)}^{t}|\epsilon(\ell)| \mathrm{d} \ell \\
& +e^{\nu\left|A_{1}\right|} \bar{\alpha} \int_{\sigma(t)}^{t}|\epsilon(s)-\epsilon(\sigma(t))| \mathrm{d} s
\end{aligned}
$$

for all $t \geq 0$, where

$$
\begin{gathered}
\bar{\zeta}_{1}=\max \left\{\nu^{2} \bar{\alpha}\left|A_{2}\right| e^{2 \nu\left|A_{1}\right|}+e^{\nu\left|A_{1}\right|}, \nu e^{\left|A_{1}\right| \nu} \bar{\alpha}\right\} \text { and } \\
\bar{\zeta}_{2}=\max \left\{\nu^{2} \bar{\alpha}\left|A_{2}\right| e^{2 \nu\left|A_{1}\right|} K_{1}, \nu e^{\left|A_{1}\right| \nu \bar{\alpha}} K_{2}\right\} .
\end{gathered}
$$

Thus,

$$
\begin{aligned}
& \left|\xi_{1}(t)-\hat{x}_{1}(t)-\xi_{\star, 1}(t)\right| \leq \nu \bar{\zeta}_{2} \sup _{\ell \in[\sigma(t), t]}|\epsilon(\ell)| \\
& +\nu \bar{\zeta}_{1} \sup _{\ell \in[\sigma(t), t]}\left(\left|\kappa_{1}(\ell)\right|+\left|\kappa_{2}(\ell)\right|\right) \\
& +\nu e^{\nu\left|A_{1}\right|} \bar{\alpha} \sup _{\ell \in[\sigma(t), t]}|\epsilon(\ell)-\epsilon(\sigma(t))|
\end{aligned}
$$

for all $t \geq \nu$.

\section{B. Proof of Theorem 1}

We introduce the variables

$$
\begin{aligned}
& x_{1}(t)=\xi_{1}(t)-\xi_{\star, 1}(t), x_{2}(t)=\xi_{2}(t)-\xi_{\star, 2}(t), \\
& \tilde{x}_{1}(t)=x_{1}(t)-\hat{x}_{1}(t), H^{\sharp}(t, s)=H\left(e^{A_{1}(t-s)}-I\right), \\
& \text { and } \epsilon^{\sharp}(t, s)=-e^{A_{1}(t-s)} \alpha(t-s)[\epsilon(t)-\epsilon(s)] .
\end{aligned}
$$

We observe that

$$
\begin{aligned}
\dot{\hat{x}}_{1}(t)= & A_{1} \hat{x}_{1}(t)+e^{A_{1}\left(t-t_{i}\right)} \alpha\left(t-t_{i}\right)\left[y(t)-y\left(t_{i}\right)\right. \\
& \left.-H\left(e^{A_{1}\left(t-t_{i}\right)}-I\right) \hat{x}_{1}\left(t_{i}\right)\right]
\end{aligned}
$$

with $y(t)=x_{2}(t)+\epsilon(t)$ and

$$
\left\{\begin{aligned}
\dot{x}_{1}(t)= & A_{1} x_{1}(t)+\kappa_{1}^{\sharp}(t) \\
\dot{x}_{2}(t)= & A_{2} x_{1}(t)+\kappa_{2}^{\sharp}(t) \\
\dot{\tilde{x}}_{1}(t)= & A_{1} \tilde{x}_{1}(t)+\epsilon^{\sharp}\left(t, t_{i}\right) \\
& -e^{A_{1}\left(t-t_{i}\right)} \alpha\left(t-t_{i}\right)\left[x_{2}(t)-x_{2}\left(t_{i}\right)\right. \\
& \left.+H\left(e^{A_{1}\left(t-t_{i}\right)}-I\right) \hat{x}_{1}\left(t_{i}\right)\right]+\kappa_{1}^{\sharp}(t) .
\end{aligned}\right.
$$

By applying variation of parameters to the $x_{1}$-subsystem in (18), then integrating the result, we obtain

$$
\begin{aligned}
& x_{2}(t)=x_{2}\left(t_{i}\right)+H\left(e^{A_{1}\left(t-t_{i}\right)}-I\right) x_{1}\left(t_{i}\right) \\
& +\int_{t_{i}}^{t} \kappa_{2}^{\sharp}(\ell) \mathrm{d} \ell+A_{2} \int_{t_{i}}^{t} \int_{t_{i}}^{m} e^{A_{1}(m-\ell)} \kappa_{1}^{\sharp}(\ell) \mathrm{d} \ell \mathrm{d} m
\end{aligned}
$$

for all $t \in\left[t_{i}, t_{i+1}\right]$. By combining (18)-(19), we get

$$
\begin{aligned}
\dot{\tilde{x}}_{1}(t)= & A_{1} \tilde{x}_{1}(t)-e^{A_{1}\left(t-t_{i}\right)} \alpha\left(t-t_{i}\right)\left[H^{\sharp}\left(t, t_{i}\right) x_{1}\left(t_{i}\right)\right. \\
& \left.-H^{\sharp}\left(t, t_{i}\right) \hat{x}_{1}\left(t_{i}\right)\right]+\epsilon^{\sharp}\left(t, t_{i}\right) \\
& -e^{A_{1}\left(t-t_{i}\right)} \alpha\left(t-t_{i}\right)\left[\int_{t_{i}}^{t} \kappa_{2}^{\sharp}(\ell) \mathrm{d} \ell\right. \\
& \left.+A_{2} \int_{t_{i}}^{t} \int_{t_{i}}^{m} e^{A_{1}(m-\ell)} \kappa_{1}^{\sharp}(\ell) \mathrm{d} \ell \mathrm{d} m\right]+\kappa_{1}^{\sharp}(t) \\
= & A_{1} \tilde{x}_{1}(t)-e^{A_{1}\left(t-t_{i}\right)} \alpha\left(t-t_{i}\right) H^{\sharp}\left(t, t_{i}\right) \tilde{x}_{1}\left(t_{i}\right) \\
& +\epsilon^{\sharp}\left(t, t_{i}\right)-e^{A_{1}\left(t-t_{i}\right)} \alpha\left(t-t_{i}\right)\left[\int_{t_{i}}^{t} \kappa_{2}^{\sharp}(\ell) \mathrm{d} \ell\right. \\
& \left.+A_{2} \int_{t_{i}}^{t} \int_{t_{i}}^{m} e^{A_{1}(m-\ell)} \kappa_{1}^{\sharp}(\ell) \mathrm{d} \ell \mathrm{d} m\right]+\kappa_{1}^{\sharp}(t) .
\end{aligned}
$$

By integrating this equation over $\left[t_{i}, t_{i+1}\right]$, we obtain

$$
\begin{aligned}
& \tilde{x}_{1}(t)=e^{A_{1}\left(t-t_{i}\right)} \tilde{x}_{1}\left(t_{i}\right)+\zeta(t) \\
& -\int_{t_{i}}^{t} e^{A_{1}(t-m)} e^{A_{1}\left(m-t_{i}\right)} \alpha\left(m-t_{i}\right) H^{\sharp}\left(m, t_{i}\right) \mathrm{d} m \tilde{x}_{1}\left(t_{i}\right)
\end{aligned}
$$


with $\zeta$ defined in (6). Thus

$$
\begin{aligned}
& \tilde{x}_{1}(t)=\zeta(t) \\
& +e^{A_{1}\left(t-t_{i}\right)}\left[I-\int_{t_{i}}^{t} \alpha\left(m-t_{i}\right) H^{\sharp}\left(m, t_{i}\right) \mathrm{d} m\right] \tilde{x}_{1}\left(t_{i}\right)
\end{aligned}
$$

and so also

$$
\begin{aligned}
& \tilde{x}_{1}(t)=\zeta(t) \\
& +e^{A_{1}\left(t-t_{i}\right)}\left[I-\int_{t_{i}}^{t} W^{\sharp}\left(m, t_{i}\right) H^{\top} H^{\sharp}\left(m, t_{i}\right) \mathrm{d} m\right] \tilde{x}_{1}\left(t_{i}\right)
\end{aligned}
$$

for all $t \in\left[t_{i}, t_{i+1}\right]$ and $i \geq 0$, where

$$
W^{\sharp}\left(m, t_{i}\right)=W\left(e^{A_{1}^{\top}\left(m-t_{i}\right)}-I\right),
$$

and where the last equality in (23) is a consequence of the definition in (5) of $\alpha$. Thus in particular, since

$$
\zeta\left(t_{i+1}\right)=0,
$$

we can specialize (23) to the case where $t=t_{i+1}$ to get

$$
\begin{aligned}
& \tilde{x}_{1}\left(t_{i+1}\right)= \\
& =e^{A_{1} \nu}\left[I-W \int_{0}^{\nu}\left(H^{\sharp}(m, 0)\right)^{\top} H^{\sharp}(m, 0) \mathrm{d} m\right] \tilde{x}_{1}\left(t_{i}\right)
\end{aligned}
$$

for all $i \in \mathbb{Z}_{\geq 0}$. The definition (4) of $W$ ensures that

$$
\tilde{x}_{1}\left(t_{i+1}\right)=0 .
$$

From this equality and (23), we deduce that for all $t \geq \nu$

$$
\tilde{x}_{1}(t)=\zeta(t) \text {. }
$$

Since $\tilde{x}_{1}=\xi_{1}-\xi_{\star, 1}-\hat{x}_{1}$, this allows us to conclude.

\section{OBSERVERS FOR Discrete MEASUREMENTS}

\section{A. Statement of Result}

In this part, we consider the case where instead of having continuous measurements of the output, the measurements are only available at discrete instants. For simplicity, we assume that the additive uncertainty $\epsilon$ on the measurements is zero, but this section can be generalized to cases where this uncertainty is nonzero. We introduce the sequence $s_{k}=k \varsigma$ for all $k \in \mathbb{Z}_{\geq 0}$ with a constant $\varsigma>0$. Consider the system

$$
\left\{\begin{aligned}
\dot{\xi}_{1}(t)= & A_{1} \xi_{1}(t)+F_{1}\left(\xi_{2}(t), u(t)\right)+\kappa_{1}(t) \\
\dot{\xi}_{2}(t)= & A_{2} \xi_{1}(t)+F_{2}\left(\xi_{2}(t), u(t)\right)+\kappa_{2}(t) \\
Y(t)= & \xi_{2}\left(s_{k}\right) \text { for all } t \in\left[s_{k}, s_{k+1}\right) \\
& \text { and } k \in \mathbb{Z}_{\geq 0}
\end{aligned}\right.
$$

with $\xi_{1}$ valued in $\mathbb{R}^{n-q}, \xi_{2}$ valued in $\mathbb{R}^{q}$, and $\kappa_{1}$ and $\kappa_{2}$ being piecewise continuous and locally bounded. As in Section II, we let $\left(A_{1}, A_{2}\right)$ be observable and $A_{1}$ be invertible. We assume:

Assumption 2: The functions $F_{1}$ and $F_{2}$ are locally Lipschitz and there are two constants $K_{1} \geq 0$ and $K_{2} \geq 0$ such that

$$
\begin{aligned}
& \left|F_{1}(a, u)-F_{1}(b, u)\right| \leq K_{1}|a-b| \text { and } \\
& \left|F_{2}(a, u)-F_{2}(b, u)\right| \leq K_{2}|a-b|
\end{aligned}
$$

for all $a \in \mathbb{R}^{q}, b \in \mathbb{R}^{q}$ and $u \in \mathbb{R}^{p}$.

Assumption 2 (which is also used in [13]) ensures that (29) is forward complete. However, the main reason why we impose Assumption 2 is that it will be needed in the proof of Theorem 2. We introduce the candidate observer:

$$
\left\{\begin{aligned}
\dot{\xi}_{\star, 1}(t)= & A_{1} \xi_{\star, 1}(t)+F_{1}(\omega(t), u(t)) \\
\dot{\xi}_{\star, 2}(t)= & A_{2} \xi_{\star, 1}(t)+F_{2}(\omega(t), u(t)) \\
\dot{\hat{x}}_{1}(t)= & A_{1} \hat{x}_{1}(t) \\
& +e^{A_{1}\left(t-t_{i}\right)} \alpha\left(t-t_{i}\right)\left[\omega(t)-\xi_{\star, 2}(t)\right. \\
& -\omega\left(t_{i}\right)+\xi_{\star, 2}\left(t_{i}\right) \\
& \left.-H\left(e^{A_{1}\left(t-t_{i}\right)}-I\right) \hat{x}_{1}\left(t_{i}\right)\right] \\
& \text { for all } t \in\left[t_{i}, t_{i+1}\right) \text { and } i \in \mathbb{Z}_{\geq 0} \\
\dot{\omega}(t)= & A_{2}\left[\hat{x}_{1}(t)+\xi_{\star, 1}(t)\right]+F_{2}(\omega(t), u(t)) \\
& \text { for all } t \in\left[s_{k}, s_{k+1}\right) \text { and } k \in \mathbb{Z}_{\geq 0} \\
\omega\left(s_{k}\right)= & \xi_{2}\left(s_{k}\right) \text { for all } k \in \mathbb{Z}_{\geq 0}
\end{aligned}\right.
$$

with $\alpha$ defined in (5) and $H=A_{2} A_{1}^{-1}$ as before, and where the solutions of (5) are defined analogously to those of (8) with $\omega(0)=0$. This observer is inspired by the one used in [6]. We also use the constant $\bar{\alpha}$ from (10), and $W$ from (4). Let us introduce the function $\beta: \mathbb{R} \rightarrow \mathbb{R}^{(n-q) \times(n-q)}$ defined by

$$
\beta(\ell)=e^{A_{1} \ell}\left[I-W \int_{0}^{\ell}\left(H^{\sharp}(m, 0)\right)^{\top} H^{\sharp}(m, 0) \mathrm{d} m\right]
$$

with $H^{\sharp}(t, s)=H\left(e^{A_{1}(t-s)}-I\right)$ as before, and the constant

$$
\bar{\beta}=|\beta|_{[0, \nu]} .
$$

In terms of our sample rates $\nu$ and $\varsigma$ for the $t_{i}$ 's and $s_{k}$ respectively, the constant $\bar{\alpha}$ from (10), and the constants

$$
\begin{gathered}
\bar{c}_{1}=\left|A_{2}\right| \max \{\bar{\beta}, 1\} e^{\left|A_{1}\right| \nu \bar{c}_{5}(\varsigma+2 \nu)+K_{2},} \\
\bar{c}_{2}=2 \max \{\bar{\beta}, 1\} \bar{c}_{5} e^{\left|A_{1}\right| \nu+\frac{\ln \left(\varsigma \bar{c}_{1}\right)}{3 \nu+\varsigma}(-6 \nu-\varsigma)}, \\
\bar{c}_{3}=2 \max \{\bar{\beta}, 1\} e^{\left|A_{1}\right| \nu \bar{c}_{5} \varsigma \frac{\nu\left|A_{2}\right| \bar{c}_{4}+1}{1-\varsigma \bar{c}_{1}}+\bar{c}_{4},} \\
\bar{c}_{4}=e^{\left|A_{1}\right| \nu} \max \left\{\nu \bar{\alpha}, \nu^{2} \bar{\alpha}|H|\left(e^{\left|A_{1}\right| \nu}-1\right)+1\right\},
\end{gathered}
$$

and

$$
\begin{aligned}
\bar{c}_{5}= & e^{\left|A_{1}\right| \nu \bar{\alpha} \nu}\left[|H| K_{1}\left(e^{\left|A_{1}\right| \nu}-1\right)+K_{2}\right] \\
& +K_{1}+2 e^{\left|A_{1}\right| \nu} \bar{\alpha},
\end{aligned}
$$

our main result of this section is:

Theorem 2: Let (29) satisfy Assumption 2 and let

$$
\varsigma \bar{c}_{1}<1
$$

hold. Then all solutions of (29) and (31) are such that

$$
\begin{aligned}
& \left|\xi_{1}(t)-\hat{x}_{1}(t)-\xi_{\star, 1}(t)\right| \\
& \leq \nu \bar{c}_{2} \sup _{m \in[0,3 \nu+\varsigma]}\left|\omega(m)-\xi_{2}(m)\right| e^{\frac{\ln \left(\varsigma \bar{c}_{1}\right)}{3 \nu+\varsigma} t} \\
& \quad+\nu \bar{c}_{3} \sup _{m \in[0, t]}\left(\left|\kappa_{1}(m)\right|+\left|\kappa_{2}(m)\right|\right)
\end{aligned}
$$

holds for all $t \geq 2 \varsigma+7 \nu$.

Remark 2: Condition (40) gives an exponential convergence rate of $-\ln \left(\varsigma \bar{c}_{1}\right) /(3 \nu+\varsigma)$, which converges to $+\infty$ as the sample rate $\varsigma$ for the sequence $\left\{s_{k}\right\}$ converges to 0 . Hence, we can ensure arbitrarily fast convergence. Condition (39) imposes a constraint on the size of the sampling interval length $\varsigma$, and $\nu$ can be chosen by the designer of the observer. 


\section{B. Proof of Theorem 2}

The proof has three parts. In the first part, we perform changes of variables that produce an error dynamics associated with the observation error. In the second step, we perform a stability analysis for the error variables. In the final step, we use the trajectory based approach from [10] and the contractivity condition from (39) to obtain the final error estimation from our theorem.

First Step. We use the new error variables

$$
\begin{aligned}
& x_{1}(t)=\xi_{1}(t)-\xi_{\star, 1}(t), x_{2}(t)=\xi_{2}(t)-\xi_{\star, 2}(t), \\
& \tilde{x}_{1}(t)=x_{1}(t)-\hat{x}_{1}(t), \text { and } r(t)=\omega(t)-\xi_{2}(t) .
\end{aligned}
$$

and the function $H^{\sharp}(t, s)=H\left(e^{A_{i}(t-s)}-I\right)$ as before. Simple calculations give

$$
\left\{\begin{aligned}
\dot{x}_{1}(t)= & A_{1} x_{1}(t)+F_{1}\left(\xi_{2}(t), u(t)\right) \\
& -F_{1}(\omega(t), u(t))+\kappa_{1}(t) \\
\dot{x}_{2}(t)= & A_{2} x_{1}(t)+F_{2}\left(\xi_{2}(t), u(t)\right) \\
& -F_{2}(\omega(t), u(t))+\kappa_{2}(t) \\
\dot{r}(t)= & A_{2} \hat{x}_{1}(t)+A_{2} \xi_{\star, 1}(t)-A_{2} \xi_{1}(t)-\kappa_{2}(t) \\
& +F_{2}(\omega(t), u(t))-F_{2}\left(\xi_{2}(t), u(t)\right) \\
& \text { for all } t \in\left[s_{k}, s_{k+1}\right) \text { and } k \in \mathbb{Z}_{\geq 0} \\
r\left(s_{k}\right)= & 0 \text { for all } k \in \mathbb{Z}_{\geq 0} .
\end{aligned}\right.
$$

Also, $x_{2}+r=\omega-\xi_{\star, 2}$. Hence

$$
\left\{\begin{aligned}
\dot{\hat{x}}_{1}(t)= & A_{1} \hat{x}_{1}(t) \\
& +e^{A_{1}\left(t-t_{i}\right)} \alpha\left(t-t_{i}\right)\left[r(t)-r\left(t_{i}\right)\right. \\
& +x_{2}(t)-x_{2}\left(t_{i}\right) \\
& \left.-H\left(e^{A_{1}\left(t-t_{i}\right)}-I\right) \hat{x}_{1}\left(t_{i}\right)\right] \\
& \text { for all } t \in\left[t_{i}, t_{i+1}\right) \text { and } i \in \mathbb{Z}_{\geq 0} \\
\dot{r}(t)= & A_{2}\left(\hat{x}_{1}(t)-x_{1}(t)\right)+F_{2}(\omega(t), u(t)) \\
& -F_{2}\left(\xi_{2}(t), u(t)\right)-\kappa_{2}(t) \\
& \text { for all } t \in\left[s_{k}, s_{k+1}\right) \text { and } k \in \mathbb{Z}_{\geq 0} \\
r\left(s_{k}\right)= & 0 \text { for all } k \in \mathbb{Z}_{\geq 0} .
\end{aligned}\right.
$$

We deduce that

$$
\begin{aligned}
\dot{\tilde{x}}_{1}(t)= & A_{1} \tilde{x}_{1}(t)-e^{A_{1}\left(t-t_{i}\right)} \alpha\left(t-t_{i}\right)\left[x_{2}(t)\right. \\
& \left.-x_{2}\left(t_{i}\right)-H\left(e^{A_{1}\left(t-t_{i}\right)}-I\right) \hat{x}_{1}\left(t_{i}\right)\right] \\
& -e^{A_{1}\left(t-t_{i}\right)} \alpha\left(t-t_{i}\right)\left[r(t)-r\left(t_{i}\right)\right]+\kappa_{1}(t) \\
& +F_{1}\left(\xi_{2}(t), u(t)\right)-F_{1}(\omega(t), u(t))
\end{aligned}
$$

By applying variation of parameters to the $\left(x_{1}, x_{2}\right)$ subsystem of (42), we obtain

$$
\begin{aligned}
x_{2}(t)= & x_{2}\left(t_{i}\right)+H\left(e^{A_{1}\left(t-t_{i}\right)}-I\right) x_{1}\left(t_{i}\right)+\psi_{1}(t) \\
& +\int_{t_{i}}^{t}\left[\kappa_{2}(\ell)+H\left(e^{A_{1}(t-\ell)}-I\right) \kappa_{1}(\ell)\right] \mathrm{d} \ell
\end{aligned}
$$

for all $t \in\left[t_{i}, t_{i+1}\right)$ and $i \geq 0$, where

$$
\begin{aligned}
\psi_{1}(t)= & H \int_{t_{i}}^{t}\left(e^{A_{1}(t-\ell)}-I\right)\left[F_{1}\left(\xi_{2}(\ell), u(\ell)\right)\right. \\
& \left.-F_{1}(\omega(\ell), u(\ell))\right] \mathrm{d} \ell+\int_{t_{i}}^{t}\left[F_{2}\left(\xi_{2}(\ell), u(\ell)\right)\right. \\
& \left.-F_{2}(\omega(\ell), u(\ell))\right] \mathrm{d} \ell .
\end{aligned}
$$

By using (45) to obtain a formula for $x_{2}(t)-x_{2}\left(t_{i}\right)$ and then replacing the $x_{2}(t)-x_{2}\left(t_{i}\right)$ in (44) by this formula, and then collecting terms, we immediately obtain

$$
\begin{aligned}
\dot{\tilde{x}}_{1}(t)= & A_{1} \tilde{x}_{1}(t)-e^{A_{1}\left(t-t_{i}\right)} \alpha\left(t-t_{i}\right) H^{\sharp}\left(t, t_{i}\right) x_{1}\left(t_{i}\right) \\
& -e^{A_{1}\left(t-t_{i}\right)} \alpha\left(t-t_{i}\right)\left[\psi_{1}(t)+\int_{t_{i}}^{t}\left[\kappa_{2}(\ell)\right.\right. \\
& \left.\left.+H^{\sharp}(t, \ell) \kappa_{1}(\ell)\right] \mathrm{d} \ell-H^{\sharp}\left(t, t_{i}\right) \hat{x}_{1}\left(t_{i}\right)\right] \\
& -e^{A_{1}\left(t-t_{i}\right)} \alpha\left(t-t_{i}\right)\left[r(t)-r\left(t_{i}\right)\right] \\
& +F_{1}\left(\xi_{2}(t), u(t)\right)-F_{1}(\omega(t), u(t))+\kappa_{1}(t) \\
= & A_{1} \tilde{x}_{1}(t)-e^{A_{1}\left(t-t_{i}\right)} \alpha\left(t-t_{i}\right) H^{\sharp}\left(t, t_{i}\right) \tilde{x}_{1}\left(t_{i}\right) \\
& +\psi_{2}(t)+\kappa_{1}(t) \\
& -e^{A_{1}\left(t-t_{i}\right)} \alpha\left(t-t_{i}\right) \int_{t_{i}}^{t} \kappa_{3}(t, \ell) \mathrm{d} \ell
\end{aligned}
$$

where $\kappa_{3}(t, \ell)=\kappa_{2}(\ell)+H^{\sharp}(t, \ell) \kappa_{1}(\ell)$ and

$$
\begin{aligned}
\psi_{2}(t)= & -e^{A_{1}(t-\sigma(t))} \alpha(t-\sigma(t)) \psi_{1}(t) \\
& -e^{A_{1}(t-\sigma(t))} \alpha(t-\sigma(t))[r(t)-r(\sigma(t))] \\
& -F_{1}(\omega(t), u(t))+F_{1}\left(\xi_{2}(t), u(t)\right) .
\end{aligned}
$$

By applying the variation of parameter to the last equality in (47) over $\left[t_{i}, t\right)$ with $t \in\left[t_{i}, t_{i+1}\right]$, we obtain

$$
\begin{aligned}
\tilde{x}_{1}(t)= & e^{A_{1}\left(t-t_{i}\right)} \tilde{x}_{1}\left(t_{i}\right) \\
& -\int_{t_{i}}^{t} e^{A_{1}(t-\ell)} e^{A_{1}\left(\ell-t_{i}\right)} \alpha\left(\ell-t_{i}\right) H^{\sharp}\left(\ell, t_{i}\right) \tilde{x}_{1}\left(t_{i}\right) \mathrm{d} \ell \\
& +\int_{t_{i}}^{t} e^{A_{1}(t-\ell)} \psi_{2}(\ell) \mathrm{d} \ell+\kappa_{\star}(t) \\
= & e^{A_{1}\left(t-t_{i}\right)}\left[I-\int_{t_{i}}^{t} \alpha\left(\ell-t_{i}\right) H^{\sharp}\left(\ell, t_{i}\right) d \ell\right] \tilde{x}_{1}\left(t_{i}\right) \\
& +\int_{t_{i}}^{t} e^{A_{1}(t-\ell)} \psi_{2}(\ell) \mathrm{d} \ell+\kappa_{\star}(t),
\end{aligned}
$$

where

$$
\begin{aligned}
& \kappa_{\star}(t)=\int_{\sigma(t)}^{t} e^{A_{1}(t-\ell)} \kappa_{1}(\ell) \mathrm{d} \ell \\
& -e^{A_{1}(t-\sigma(t))} \int_{\sigma(t)}^{t} \alpha(m-\sigma(t)) \int_{\sigma(t)}^{m} \kappa_{3}(t, \ell) \mathrm{d} \ell \mathrm{d} m .
\end{aligned}
$$

From the definition of $\alpha$ in (5), we get

$$
\begin{aligned}
& \tilde{x}_{1}(t)=\int_{t_{i}}^{t} e^{A_{1}(t-\ell)} \psi_{2}(\ell) \mathrm{d} \ell+\kappa_{\star}(t) \\
& +e^{A_{1}\left(t-t_{i}\right)}\left[I-\int_{t_{i}}^{t} W\left(H^{\sharp}\left(\ell, t_{i}\right)\right)^{\top} H^{\sharp}\left(\ell, t_{i}\right) \mathrm{d} \ell\right] \tilde{x}_{1}\left(t_{i}\right)
\end{aligned}
$$

for all $t \in\left[t_{i}, t_{i+1}\right]$ and all $i \geq 0$.

Thus, we deduce from the definition (4) of $W$ that

$$
\begin{aligned}
\tilde{x}_{1}\left(t_{i+1}\right) & =\int_{t_{\dot{t}}}^{t_{i+1}} e^{A_{1}\left(t_{i+1}-\ell\right)} \psi_{2}(\ell) \mathrm{d} \ell+\kappa_{\star}\left(t_{i+1}\right) \\
& =\int_{t_{i}}^{t_{i+1}} e^{A_{1}\left(t_{i+1}-\ell\right)} \psi_{2}(\ell) \mathrm{d} \ell
\end{aligned}
$$

for all $i \geq 0$. Combining (51) and (50), we deduce that

$$
\begin{aligned}
& \tilde{x}_{1}(t)=\int_{t_{i}}^{t} e^{A_{1}(t-\ell)} \psi_{2}(\ell) \mathrm{d} \ell+\kappa_{\star}(t) \\
& +e^{A_{1}\left(t-t_{i}\right)}\left[I-\tilde{W}\left(t-t_{i}\right)\right] \int_{t_{i-1}}^{t_{i}} e^{A_{1}\left(t_{i}-\ell\right)} \psi_{2}(\ell) \mathrm{d} \ell
\end{aligned}
$$

for all $t \geq \nu$, where

$$
\tilde{W}(s)=W \int_{0}^{s}\left(H^{\sharp}(\ell, 0)\right)^{\top} H^{\sharp}(\ell, 0) \mathrm{d} \ell
$$

This gives

$$
\left\{\begin{aligned}
\tilde{x}_{1}(t)= & \beta(t-\sigma(t)) \int_{\sigma(t)-\nu}^{\sigma(t)} e^{A_{1}(\sigma(t)-\ell)} \psi_{2}(\ell) \mathrm{d} \ell \\
& +\int_{\sigma(t)}^{t} e^{A_{1}(t-\ell)} \psi_{2}(\ell) \mathrm{d} \ell+\kappa_{\star}(t) \\
\dot{r}(t)= & -A_{2} \tilde{x}_{1}(t)+F_{2}(\omega(t), u(t)) \\
& -F_{2}\left(\xi_{2}(t), u(t)\right)-\kappa_{2}(t) \\
& \text { for all } t \in\left[s_{k}, s_{k+1}\right) \text { and } k \in \mathbb{Z}_{\geq 0} \\
r\left(s_{k}\right)= & 0 \text { for all } k \in \mathbb{Z}_{\geq 0}
\end{aligned}\right.
$$

with $\beta$ defined in (32) for all $t \geq \nu$. 
Second Step. Now, we perform a stability analysis of the system (54). Integrating the second equality in (54) over $\left[s_{k}, t\right)$ with $t \in\left[s_{k}, s_{k+1}\right)$, we obtain

$$
\begin{aligned}
r(t)= & -A_{2} \int_{s_{k}}^{t} \tilde{x}_{1}(m) \mathrm{d} m-\int_{s_{k}}^{t} \kappa_{2}(m) \mathrm{d} m \\
& +\int_{s_{k}}^{t}\left[F_{2}(\omega(m), u(m))\right. \\
& \left.-F_{2}\left(\xi_{2}(m), u(m)\right)\right] \mathrm{d} m
\end{aligned}
$$

for all $k \in \mathbb{Z}_{\geq 0}$. Consequently,

$$
\begin{aligned}
|r(t)| \leq & \left|A_{2}\right| \int_{s_{k}}^{t}\left|\tilde{x}_{1}(m)\right| \mathrm{d} m+K_{2} \int_{s_{k}}^{t}|r(m)| \mathrm{d} m \\
& \int_{s_{k}}^{t}\left|\kappa_{2}(m)\right| \mathrm{d} m
\end{aligned}
$$

for all $t \in\left[s_{k}, s_{k+1}\right]$. On the other hand, (54) gives

$$
\begin{aligned}
\left|\tilde{x}_{1}(t)\right| \leq & \bar{\beta} \int_{\sigma(t)-\nu}^{\sigma(t)} e^{\left|A_{1}\right| \nu}\left|\psi_{2}(\ell)\right| \mathrm{d} \ell \\
& +\int_{\sigma(t)}^{t} e^{\left|A_{1}\right| \nu}\left|\psi_{2}(\ell)\right| \mathrm{d} \ell+\left|\kappa_{\star}(t)\right| \\
\leq & \max \{\bar{\beta}, 1\} e^{\left|A_{1}\right| \nu} \int_{\sigma(t)-\nu}^{t}\left|\psi_{2}(\ell)\right| \mathrm{d} \ell \\
& +\left|\kappa_{\star}(t)\right| .
\end{aligned}
$$

for all $t \geq 0$. The last inequality and (56) give

$|r(t)| \leq$

$\left|A_{2}\right| \max \{\bar{\beta}, 1\} e^{\left|A_{1}\right| \nu} \int_{s_{k}}^{t} \int_{\sigma(m)-\nu}^{m}\left|\psi_{2}(\ell)\right| \mathrm{d} \ell \mathrm{d} m$

$+\left|A_{2}\right| \int_{s_{k}}^{t}\left|\kappa_{\star}(m)\right| \mathrm{d} m+\int_{s_{k}}^{t}\left(K_{2}|r(m)|+\left|\kappa_{2}(m)\right|\right) \mathrm{d} m$.

As an immediate consequence,

$$
\begin{aligned}
& |r(t)| \leq \varsigma\left|A_{2}\right| \max \{\bar{\beta}, 1\} e^{\left|A_{1}\right| \nu} \int_{t-\varsigma-2 \nu}^{t}\left|\psi_{2}(\ell)\right| \mathrm{d} \ell \\
& +\int_{t-\varsigma}^{t}\left(K_{2}|r(m)|+\left|A_{2}\right|\left|\kappa_{\star}(m)\right|+\left|\kappa_{2}(m)\right|\right) \mathrm{d} m
\end{aligned}
$$

for all $t \geq \varsigma+2 \nu$. Also, Assumption 2 implies that

$$
\begin{aligned}
\left|\psi_{1}(t)\right| \leq & |H| K_{1} \int_{\sigma(t)}^{t}\left|e^{A_{1}(t-\ell)}-I\right||r(\ell)| \mathrm{d} \ell \\
& +K_{2} \int_{\sigma(t)}^{t}|r(\ell)| \mathrm{d} \ell \text { and } \\
\left|\psi_{2}(t)\right| \leq & e^{\left|A_{1}\right| \nu} \bar{\alpha}\left|\psi_{1}(t)\right|+e^{\left|A_{1}\right| \nu} \bar{\alpha}\left|r(t)-r\left(t_{i}\right)\right| \\
& +K_{1}|r(t)| .
\end{aligned}
$$

Thus, with the choice

$$
K^{\sharp}(s)=K_{1}|H|\left|e^{A_{1} s}-I\right|+K_{2},
$$

we get

$$
\begin{aligned}
\left|\psi_{2}(t)\right| \leq & e^{\left|A_{1}\right| \nu} \bar{\alpha}|H| K_{1} \int_{\sigma(t)}^{t}\left|e^{A_{1}(t-\ell)}-I\right||r(\ell)| \mathrm{d} \ell \\
& +e^{\left|A_{1}\right| \nu} \bar{\alpha} K_{2} \int_{\sigma(t)}^{t}|r(\ell)| \mathrm{d} \ell \\
& +e^{\left|A_{1}\right| \nu} \bar{\alpha}|r(t)-r(\sigma(t))|+K_{1}|r(t)| \\
\leq & e^{\left|A_{1}\right| \nu \bar{\alpha} \int_{\sigma(t)}^{t} K^{\sharp}(t-\ell)|r(\ell)| \mathrm{d} \ell} \\
& +e^{\left|A_{1}\right| \nu} \bar{\alpha}|r(\sigma(t))|+\left(K_{1}+e^{\left|A_{1}\right| \nu} \bar{\alpha}\right)|r(t)| \\
\leq & \bar{c}_{5}|r|_{[t-\nu, t]}
\end{aligned}
$$

with $\bar{c}_{5}$ defined in (38). Combining (62) with (59), we obtain

$$
\begin{aligned}
& |r(t)| \leq \int_{t-\varsigma}^{t}\left(\left|A_{2}\right|\left|\kappa_{\star}(m)\right|+\left|\kappa_{2}(m)\right|\right) \mathrm{d} m \\
& +\varsigma\left|A_{2}\right| \max \{\bar{\beta}, 1\} e^{\left|A_{1}\right| \nu \int_{t-\varsigma-2 \nu}^{t} \bar{c}_{5}|r|_{[\ell-\varsigma, \ell]} \mathrm{d} \ell} \\
& +K_{2} \int_{t-\varsigma}^{t}|r(m)| \mathrm{d} m
\end{aligned}
$$

for all $t \geq \varsigma+3 \nu$. Hence, with $\bar{c}_{1}$ as defined in (39), we have

$$
\begin{aligned}
& |r(t)| \leq \varsigma\left|A_{2}\right| \max \{\bar{\beta}, 1\} e^{\left|A_{1}\right| \nu \bar{c}_{5}(\varsigma+2 \nu)|r|_{[t-\varsigma-3 \nu, t]}} \\
& +K_{2} \varsigma|r|_{[t-\varsigma-3 \nu, t]} \\
& +\int_{t-\varsigma}^{t}\left(\left|A_{2}\right|\left|\kappa_{\star}(m)\right|+\left|\kappa_{2}(m)\right|\right) \mathrm{d} m \\
& \leq \varsigma\left[\left|A_{2}\right| \max \{\bar{\beta}, 1\} e^{\left|A_{1}\right| \nu \bar{c}_{5}(\varsigma+2 \nu)}\right. \\
& \left.+K_{2}\right]|r|_{[t-\varsigma-3 \nu, t]} \\
& +\int_{t-\varsigma}^{t}\left(\left|A_{2}\right|\left|\kappa_{\star}(m)\right|+\left|\kappa_{2}(m)\right|\right) \mathrm{d} m \\
& \leq \varsigma \bar{c}_{1}|r|_{[t-\varsigma-3 \nu, t]} \\
& +\int_{t-\varsigma}^{t}\left(\left|A_{2}\right|\left|\kappa_{\star}(m)\right|+\left|\kappa_{2}(m)\right|\right) \mathrm{d} m .
\end{aligned}
$$

From the definition of $\kappa_{\star}$ in (49), we deduce that

$$
\begin{aligned}
& \left|\kappa_{\star}(t)\right| \leq \bar{\alpha} e^{\left|A_{1}\right| \nu} \int_{\sigma(t)}^{t}\left[\int_{\sigma(t)}^{m}\left|\kappa_{2}(\ell)\right| \mathrm{d} \ell\right. \\
& \left.+\int_{\sigma(t)}^{m}|H| \int_{\sigma(t)}^{s}\left|e^{A_{1}(s-\ell)}-I\right|\left|\kappa_{1}(\ell)\right| \mathrm{d} \ell\right] \mathrm{d} s \\
& +e^{\left|A_{1}\right| \nu} \int_{\sigma(t)}^{t}\left|\kappa_{1}(\ell)\right| \mathrm{d} \ell \\
& \leq \nu \bar{\alpha} e^{\left|A_{1}\right| \nu}\left[\int_{\sigma(t)}^{t}\left|\kappa_{2}(\ell)\right| \mathrm{d} \ell\right. \\
& \left.+\int_{\sigma(t)}^{t}|H| \int_{\sigma(t)}^{s}\left(e^{\left|A_{1}\right|(s-\ell)}-1\right)\left|\kappa_{1}(\ell)\right| \mathrm{d} \ell \mathrm{d} s\right] \\
& +e^{\left|A_{1}\right| \nu} \int_{\sigma(t)}^{t}\left|\kappa_{1}(\ell)\right| \mathrm{d} \ell \\
& \leq \nu \bar{\alpha} e^{\left|A_{1}\right| \nu} \int_{\sigma(t)}^{t}\left(\left|\kappa_{2}(\ell)\right|\right. \\
& \left.+\nu|H|\left(e^{\left|A_{1}\right| \nu}-1\right)\left|\kappa_{1}(\ell)\right|\right) \mathrm{d} \ell \\
& +e^{\left|A_{1}\right| \nu} \int_{\sigma(t)}^{t}\left|\kappa_{1}(\ell)\right| \mathrm{d} \ell \\
& =\int_{\sigma(t)}^{t}\left[\nu \bar{\alpha} e^{\left|A_{1}\right| \nu}\left|\kappa_{2}(\ell)\right|+e^{\left|A_{1}\right| \nu} \nu^{\sharp}\left|\kappa_{1}(\ell)\right|\right] \mathrm{d} \ell \\
& \leq \bar{c}_{4} \int_{\sigma(t)}^{t}\left[\kappa_{1}(\ell)|+| \kappa_{2}(\ell) \mid\right] \mathrm{d} \ell
\end{aligned}
$$

where $\nu^{\sharp}=\nu^{2} \bar{\alpha}|H| e^{\left|A_{1}\right| \nu}-\nu^{2} \bar{\alpha}|H|+1$ and $\bar{c}_{4}$ is from (37). Thus, with the choice $\tilde{\kappa}(\ell)=\left|\kappa_{1}(\ell)\right|+\left|\kappa_{2}(\ell)\right|$, we have

$$
\begin{aligned}
|r(t)| \leq & \varsigma \bar{c}_{1}|r|_{[t-\varsigma-3 \nu, t]} \\
& +\int_{t-\varsigma}^{t}\left(\left|A_{2}\right| \bar{c}_{4} \int_{\sigma(m)}^{m} \tilde{\kappa}(\ell) \mathrm{d} \ell+\left|\kappa_{2}(m)\right|\right) \mathrm{d} m \\
\leq & \varsigma \bar{c}_{1}|r|_{[t-\varsigma-3 \nu, t]}+\varsigma \nu\left|A_{2}\right| \bar{c}_{4}|\tilde{\kappa}|_{[t-\nu-\varsigma, t]} \tilde{\kappa}(\ell) \\
& +\varsigma\left|\kappa_{2}\right|[t-\nu-\varsigma, t] \\
\leq & \varsigma \bar{c}_{1}|r|_{[t-\varsigma-3 \nu, t]}+\varsigma\left(\nu\left|A_{2}\right| \bar{c}_{4}+1\right)|\tilde{\kappa}|_{[t-\nu-\varsigma, t]}
\end{aligned}
$$

for all $t \geq \varsigma+3 \nu$.

Third Step. We now apply the trajectory based contractivity method from [13, Lemma 1] to the function $w(t)=$ $|r(t+\varsigma+3 \nu)|$. This gives

$$
\begin{aligned}
|r(t)| \leq & \sup _{m \in[0, \varsigma+3 \nu]}|r(m)| e^{\frac{\ln \left(\varsigma \bar{c}_{1}\right)}{3 \nu+\varsigma}(t-3 \nu-\varsigma)} \\
& +\varsigma \frac{\nu\left|A_{2}\right| \bar{c}_{4}+1}{1-\varsigma \bar{c}_{1}} \sup _{m \in[0, t]} \tilde{\kappa}(m)
\end{aligned}
$$

for all $t \geq \varsigma+3 \nu$. We deduce from (54) and (62) that

$$
\begin{aligned}
\left|\tilde{x}_{1}(t)\right| \leq & \bar{\beta} \int_{\sigma(t)-\nu}^{\sigma(t)} e^{\left|A_{1}\right| \nu}\left|\psi_{2}(\ell)\right| \mathrm{d} \ell \\
& +\int_{\sigma(t)}^{t} e^{\left|A_{1}\right| \nu}\left|\psi_{2}(\ell)\right| \mathrm{d} \ell+\left|\kappa_{\star}(t)\right| \\
\leq & \max \{\bar{\beta}, 1\} e^{\left|A_{1}\right| \nu} \int_{t-2 \nu}^{t}\left|\psi_{2}(\ell)\right| \mathrm{d} \ell+\left|\kappa_{\star}(t)\right| \\
\leq & \max \{\bar{\beta}, 1\} e^{\left|A_{1}\right| \nu} \int_{t-2 \nu}^{t} \bar{c}_{5}|r|_{[\ell-\nu, \ell]} \mathrm{d} \ell \\
& +\left|\kappa_{\star}(t)\right| \\
\leq & \bar{c}_{6} \nu|r|_{[t-3 \nu, t]}+\left|\kappa_{\star}(t)\right|
\end{aligned}
$$

for all $t \geq \varsigma+4 \nu$, where $\bar{c}_{6}=2 \max \{\bar{\beta}, 1\} e^{\left|A_{1}\right| \nu \bar{c}_{5}}$. 
Combining this inequality with (67) and (65), we obtain

$$
\begin{aligned}
& \left|\tilde{x}_{1}(t)\right| \leq \bar{c}_{4} \int_{\sigma(t)}^{t}\left[\kappa_{1}(\ell)|+| \kappa_{2}(\ell) \mid\right] \mathrm{d} \ell \\
& +\bar{c}_{6} \nu \sup _{\ell \in[t-3 \nu, t]}\left[\sup _{m \in[0, \varsigma+3 \nu]}|r(m)| e^{\frac{\ln \left(\varsigma \bar{c}_{1}\right)}{3 \nu+\varsigma}(\ell-3 \nu-\varsigma)}\right. \\
& \left.+\varsigma \frac{\nu\left|A_{2}\right| \bar{c}_{4}+1}{1-\varsigma \bar{c}_{1}} \sup _{m \in[0, \ell]}\left(\left|\kappa_{1}(m)\right|+\left|\kappa_{2}(m)\right|\right)\right]
\end{aligned}
$$

for all $t \geq 2 \varsigma+7 \nu$. It follows that

$$
\begin{aligned}
& \left|\tilde{x}_{1}(t)\right| \leq \\
& 2 \max \{\bar{\beta}, 1\} e^{\left|A_{1}\right| \nu} \bar{c}_{5} \nu|r|_{[0, \varsigma+3 \nu]} e^{\frac{\ln \left(\varsigma \bar{c}_{1}\right)}{3 \nu+\varsigma}(t-6 \nu-\varsigma)} \\
& +\nu\left[2 \max \{\bar{\beta}, 1\} e^{\left|A_{1}\right| \nu} \bar{c}_{5} \varsigma \frac{\nu\left|A_{2}\right| \bar{c}_{4}+1}{1-\varsigma \bar{c}_{1}}+\bar{c}_{4}\right]|\tilde{\kappa}|_{[0, t]} .
\end{aligned}
$$

This allows us to conclude the proof.

\section{ILLUSTRATIONS}

As in [11], we first study a pendulum model

$$
\left\{\begin{aligned}
\dot{a}_{1}(t) & =a_{2}(t) \\
\dot{a}_{2}(t) & =-\sin \left(a_{1}(t)\right) \\
z(t) & =a_{1}\left(s_{j}\right) \text { if } t \in\left[s_{j}, s_{j+1}\right)
\end{aligned}\right.
$$

with the $a_{i}$ 's valued in $\mathbb{R}$.

The change of coordinates given by $\xi_{1}(t)=a_{1}(t)+a_{2}(t)$ and $\xi_{2}(t)=a_{1}(t)$ transforms the system (71) into

$$
\left\{\begin{array}{l}
\dot{\xi}_{1}(t)=\xi_{1}(t)-\xi_{2}(t)-\sin \left(\xi_{2}(t)\right) \\
\dot{\xi}_{2}(t)=\xi_{1}(t)-\xi_{2}(t) \\
y(t)=\xi_{2}\left(s_{j}\right) \text { if } t \in\left[s_{j}, s_{j+1}\right)
\end{array}\right.
$$

which is covered by Theorem 2 .

Then, with the notation of the previous section, we take $A_{1}=A_{2}=1, F_{1}\left(\xi_{2}\right)=-\xi_{2}-\sin \left(\xi_{2}\right)$ and $F_{2}\left(\xi_{2}\right)=-\xi_{2}$, $K_{1}=2$, and $K_{2}=1$. Simple calculations show that in this case, the function $\alpha$ that we defined in (5) is

$$
\alpha(m)=\frac{e^{m}-1}{\frac{e^{2 \nu}-1}{2}-2\left(e^{\nu}-1\right)+\nu}
$$

and therefore also the observer

$$
\left\{\begin{aligned}
\dot{\xi}_{\star, 1}(t)= & \xi_{\star, 1}(t)-\omega(t)-\sin (\omega(t)) \\
\dot{\xi}_{\star, 2}(t)= & \xi_{\star, 1}(t)-\omega(t) \\
\dot{\hat{x}}_{1}(t)= & \hat{x}_{1}(t) \\
& +\frac{2 e^{t-t_{i}}\left(e^{t-t_{i}}-1\right)}{e^{2 \nu}-1-4\left(e^{\nu}-1\right)+2 \nu}\left[\omega(t)-\omega\left(t_{i}\right)\right. \\
& -\xi_{\star, 2}(t)+\xi_{\star, 2}\left(t_{i}\right) \\
& \left.-\left(e^{\left(t-t_{i}\right)}-I\right) \hat{x}_{1}\left(t_{i}\right)\right] \\
& \text { for all } t \in\left[t_{i}, t_{i+1}\right) \text { and } i \in \mathbb{Z}_{\geq 0} \\
\dot{\omega}(t)= & \hat{x}_{1}(t)+\xi_{\star, 1}(t)-\omega(t) \\
& \text { for all } t \in\left[s_{k}, s_{k+1}\right) \text { and } k \in \mathbb{Z}_{\geq 0} \\
\omega\left(s_{k}\right)= & \xi_{2}\left(s_{k}\right) \text { for all } k \in \mathbb{Z}_{\geq 0}
\end{aligned}\right.
$$

which differs from the observers in [11] which have delays.

We can also apply Theorem 1 to design observers for the single link robotic manipulator dynamics in [11], to achieve a similar advantage of having observers that are free of delay terms while providing a fixed convergence time (which is a stronger result than the finite time convergence result in [18]), but without requiring the use of delayed output measurements that were required in [11].

\section{CONCLUSiON}

We provided a new class of observers for which the finite convergence time of the observer value to the true unknown state value is independent of the initial state, when continuous measurements of the output are available. By not requiring delayed output values in the observer, and by providing a reduced order observer structure, our methods may offer computational advantages as compared with earlier methods. Our methods apply to a a family of continuoustime systems, and are based on the observability Gramian. We adapted this observer to cases where only discrete time measurements are available, where one instead obtains arbitrarily fast convergence. Extensions including new robustness results can be proved and time-varying version are expected.

\section{REFERENCES}

[1] S. Ahmed, M. Malisoff, and F. Mazenc. Finite time estimation for time-varying systems with delay in the measurements. Systems and Control Letters, 133:104551, 2019.

[2] F. Cacace, A. Germani, and C. Manes. An observer for a class of nonlinear systems with time varying observation delay. Systems and Control Letters, 59:305-312, 2010.

[3] F. Cacace, A. Germani, and C. Manes. A new approach to design interval observers for linear systems. IEEE Transactions on Automatic Control, 60(6):1665-1670, 2015.

[4] R. Engel and G. Kreisselmeier. A continuous time observer which converges in finite time. IEEE Transactions on Automatic Control, 47(7):1202-1204, 2002.

[5] J. Holloway and M. Krstic. Prescribed-time observers for linear systems in observer canonical form. IEEE Transactions on Automatic Control, 64(9):3905-3912, 2019.

[6] I. Karafyllis and C. Kravaris. From continuous-time design to sampleddata design of observers. IEEE Transactions on Automatic Control, 54(9):2169-2174, 2009.

[7] Y. Li and R. Sanfelice. A finite-time convergent observer with robustness to piecewise-constant measurement noise. Automatica, 57:222-230, 2015.

[8] D. Luenberger. Introduction to Dynamic Systems. John Wiley and Sons, New York, 1979.

[9] F. Mazenc, E. Fridman, and W. Djema. Estimation of solutions of observable nonlinear systems with disturbances. Systems and Control Letters, 79:47-58, 2015.

[10] F. Mazenc and M. Malisoff. Trajectory based approach for the stability analysis of nonlinear systems with time delays. IEEE Transactions on Automatic Control, 60(6):1716-1721, 2015.

[11] F. Mazenc, M. Malisoff, and Z.P. Jiang. Reduced order fast converging observer for systems with discrete measurements and sensor noise. Systems and Control Letters, 2021, to appear.

[12] F. Mazenc, M. Malisoff, and S. Niculescu. Sampled-data estimator for nonlinear systems with arbitrarily fast rate of convergence. In Proc. American Control Conference, pages 1685-1689, Denver, CO, 2020.

[13] F. Mazenc, M. Malisoff, and S-I. Niculescu. Stability and control design for time-varying systems with time-varying delays using a trajectory-based approach. SIAM Journal on Control and Optimization, 55(1):533-556, 2017.

[14] T. Menard, E. Moulay, and W. Perruquetti. A global high-gain finite time observer. IEEE Transactions on Automatic Control, 55(6):1500_ 1506,2010

[15] T. Raff and F. Allgower. An observer that converges in finite time due to measurement-based state updates. IFAC Proceedings Volumes, 41(2):2693-2695, 2008.

[16] F. Sauvage, M. Guay, and D. Dochain. Design of a nonlinear finite time converging observer for a class of nonlinear systems. Journal of Control Science and Engineering, 2007(36954):9pp., 2007.

[17] H. Silm, R. Ushirobira, D. Efimov, J. Richard, and W. Michiels. A note on distributed finite-time observers. IEEE Transactions on Automatic Control, 64(2):759-766, 2019

[18] Z-L. Zhao and Z.P. Jiang. Semi-global finite-time output-feedback stabilization with an application to robotics. IEEE Transactions on Industrial Electronics, 66(4):3148-3156, 2019. 